\title{
Descripción Anatómica de la Inervación del Miembro Pélvico de León Africano (Panthera leo)
}

\author{
Anatomical Description of Pelvic Limb Innervation of African Lion (Panthera leo)
}

\author{
Rodemil Medina Puentes*; Pamela Morales Muñoz*; Ismael Concha Albornoz ${ }^{*}$ \& Cintya Borroni González**
}

\begin{abstract}
MEDINA, P. R.; MORALES, M. P.; CONCHA, A. I. \& BORRONI, G. C. Descripción anatómica de la inervación del miembro pélvico de león africano (Panthera leo). Int. J. Morphol., 32(3):889-894, 2014.

RESUMEN: El león africano (Panthera leo) pertenece a la familia felidae del orden carnívora, corresponde a un depredador de gran tamaño, en Chile se encuentra únicamente en estado de cautiverio, sin embargo está presente en numerosos zoológicos. La preocupación e interés por el bienestar de estos animales ha aumentado, por lo cual, cada vez se realizan procedimientos médicos de mayor complejidad. Lo expuesto anteriormente genera la necesidad de profundizar los conocimientos anatómicos que existen de esta especie. Por lo anterior, el objetivo de este trabajo fue realizar un estudio anatómico detallado de la inervación del miembro pélvico del león y describir sus relaciones topográficas con el sistema músculo esquelético y vascular. Se disecó un cadáver de león hembra, adulta, presentando la descripción según las regiones topográficas: región del cíngulo miembro pélvico, región femoral, región crural y región del pie. Se realizó un registro fotográfico de las disecciones, el cual fue complementada con ilustraciones anatómicas representativas de cada región. Acorde a lo observado, podemos indicar que existe una gran similitud con lo descrito en el gato, destacando el gran desarrollo de los nervios y ramos musculares del león. La presente descripción, permite, ampliar el conocimiento de la anatomía del miembro pélvico del león africano, específicamente su inervación y relaciones musculares y vasculares.
\end{abstract}

PALABRAS CLAVE: León africano; Nervio isquiático; Inervación; Miembro pélvico.

\section{INTRODUCCIÓN}

El león africano (Panthera leo) pertenece a la familia felidae del orden carnívora, una de las cuatro especies del género Panthera; es el segundo felino más grande después del tigre y puede alcanzar un peso entre $150-250 \mathrm{~kg}$. Habita normalmente en África y Asia, pero en Chile se encuentra únicamente en estado de cautiverio. Está presente en los zoológicos de todo el mundo y consideramos que esta especie es muy importante para los zoológicos y centros de rescate de felinos, por lo que la preocupación e interés por estos animales se ha incrementado, y cada vez se realizan procedimientos de mayor complejidad.

Los miembros pélvicos son frecuentemente abordados en procedimientos médicos de distinto orden, como en la utilización de sus vías venosas para administración de fármacos, ortopedia, y lugar de administración de dardos tranquilizantes. En la realización de estos procedimientos se ha tomado como guía la anatomía del gato doméstico, sin tener certeza de las similitudes o diferencias que pudiesen existir entre éste y el león. Por lo tanto, es útil conocer en detalle la anatomía del área, especialmente de las estructu- ras más delicadas y de importancia crítica en procedimientos complejos; como los nervios y la vasculatura, su respectivo recorrido y relaciones topográficas. Lo expuesto anteriormente genera la necesidad de profundizar los conocimientos anatómicos que existen de esta especie.

\section{MATERIAL Y MÉTODO}

Para el estudio, se utilizó un cadáver de león africano hembra adulta de $130 \mathrm{~kg}$, donada por el Zoológico Metropolitano. El cadáver se fijó y conservó en la unidad de Anatomía Veterinaria de la Universidad Santo Tomás, sede Santiago, posteriormente se realizó repleción arterio-venosa con látex natural pigmentado. Para la disección de los miembros pélvicos se utilizó instrumental de disección de rutina.

El presente trabajo corresponde a un estudio anatómico de tipo descriptivo de la inervación del miembro pélvico del león y sus relaciones topográficas. La disección

\footnotetext{
* Escuela de Medicina Veterinaria. Facultad de Medicina Veterinaria y Recursos Naturales. Universidad Santo Tomás, Chile.

***Escuela de Medicina Veterinaria. Facultad de Ecología y Recursos Naturales. Universidad Andrés Bello, Chile.
} 
se realizó de proximal a distal describiendo la inervación y relaciones topográficas de las siguientes regiones anatómicas: región del cíngulo miembro pélvico; región femoral; región crural, y región del pie. En cada una de las disecciones se procedió a realizar registros fotográficos y además se realizaron ilustraciones anatómicas originales que complementan la descripción.

Para comenzar la disección muscular y la visualización de la inervación y vasculatura, se extrajo el tegumento del miembro pélvico izquierdo y derecho. Posteriormente, se realizaron incisiones en las fascias profundas del miembro, éstas se rechazaron para observar y disecar los músculos mediante divulsión del tejido conectivo entre ellos. Se realizó la exposición de la región pélvica para observar el tronco lumbosacro, para esto se realizó una incisión en los vientres de los músculos glúteos. Para observar el nervio isquiático se procedió a desinsertar distalmente el músculo bíceps femoral.

Se procedió a disecar la vasculatura y nervios de la región femoral, profundo al músculo bíceps femoral. Luego se continuó con la disección de la región crural, quedando visibles los nervios fibular superficial, nervio fibular profundo y nervio tibial.

\section{RESULTADOS Y DISCUSIÓN}

Región del cíngulo miembro pélvico. En la región pélvica se observó el tronco lumbosacro, emergiendo por medial al ala del ilion, entre los músculos piriforme y glúteo profundo, de inmediato da origen al nervio glúteo craneal, el cual origina ramos para el músculo glúteo medio y $\mathrm{m}$. glúteo profundo, y un pequeño ramo al músculo piriforme (Fig. 1). Se observó también un ramo al $\mathrm{m}$. tensor de la fascia lata, que cursa superficialmente al m. glúteo profundo. Igual distribución se describe en el perro (Evans, 2013).

Ligeramente caudal y dorsal a los nervios mencionados, se puede apreciar la participación de los ramos ventrales de los nervios sacros (S1, S2 y S3) en la formación del tronco lumbosacro, a diferencia de lo descrito en el gato donde participa S1 y una pequeña participación de S2 (Fig. 1) (Ghoshal, 1982).

El nervio glúteo caudal, se dividió en tres ramos para el músculo glúteo superficial y tres para el m. glúteofemoral. El nervio glúteo caudal en el perro inerva los mismos músculos con excepción del glúteofemoral que no está presente (Evans).

Originándose de la parte sacra del plexo lumbosacro, emergen dos nervios, el nervio pudendo que se divide en un ramo perineal y otro genital, y el nervio femoral cutáneo caudal. Ambos pasan por medial a las ramas musculares de la arteria y vena glútea caudal, y al músculo abductor crural caudal. También originándose del plexo sacro se pudo observar un ramo al músculo coxígeo y $\mathrm{m}$. elevador del ano. Por último, el principal ramo del tronco lumbosacro es el nervio isquiático, el cual se describirá en la región femoral (Fig. 1).

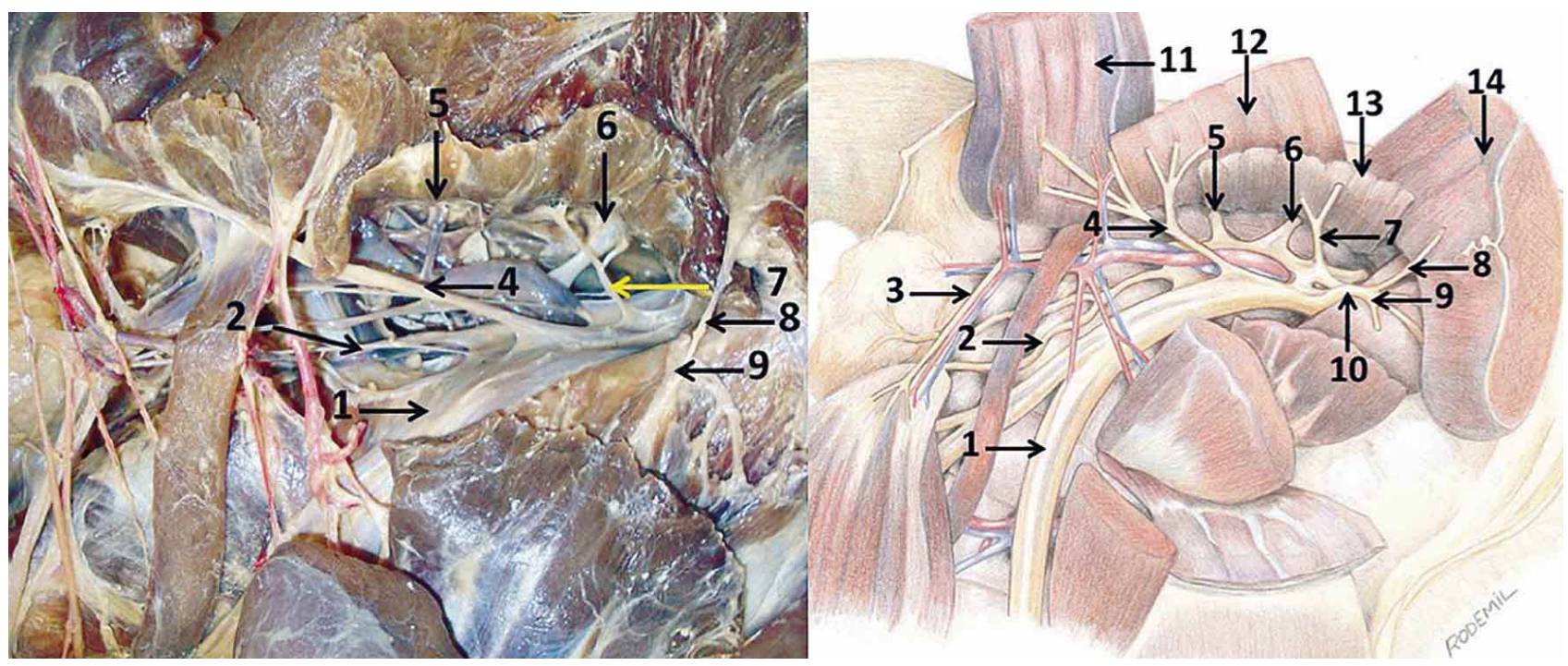

Fig. 1. Imagen y esquema de la región del cíngulo miembro pélvico de león. Miembro pélvico derecho, vista lateral. Dibujo original Rodemil Medina. $1=\mathrm{N}$. isquiático. 2=N. pudendo. 3= N. cutáneo femoral caudal. 4= N. glúteo caudal. 5= Ramo ventral de $S 3$. $6=$ Ramo ventral de S2. 7= Ramos al m. piriforme. 8= Ramos al m. glúteo medio. 9= Ramos al m. glúteo profundo. 10= N. glúteo craneal. 11= M. glúteo femoral. 12= M. glúteo superficial. 13= M. piriforme. 14= M. glúteo medio. 
Región femoral. El nervio isquiático, cursa por proximal y luego por caudal al trocánter mayor del fémur, en profundidad a los músculos glúteofemoral y bíceps femoral, y superficialmente a la cara lateral de los músculos gemelo caudal y cuadrado femoral. En su tercio proximal, proporciona varios ramos musculares: dos ramos al $\mathrm{m}$. bíceps femoral, un ramo que se divide hacia el $\mathrm{m}$. bíceps femoral y al $\mathrm{m}$. semitendinoso y un ramo al m. semimembranoso (Fig. 2B); éstos se observan parcialmente cubiertos por el músculo abductor crural caudal, el que es inervado mediante un pequeño ramo distal a los anteriores. En el tercio medio del nervio isquiático se originan los nervios cutáneo sural caudal proximal y el nervio cutáneo sural lateral (Fig. 2B), que perforan y atraviesan al $\mathrm{m}$. bíceps femoral. Por último, en su tercio distal, el nervio isquiático se encuentra entre el $\mathrm{m}$. bíceps femoral y la cara lateral del músculo semimembranoso, aquí se divide en sus ramos terminales, el nervio fibular común y nervio tibial, los cuales están separados por la arteria y vena femoral caudal distal, que cruzan craneocaudalmente, por lateral al nervio tibial y medial al nervio fibular común (Fig. 2A y 2B). Estas relaciones topográficas ocurren también en el gato, según las imágenes obtenidas por Done (2002). Además, Evans y Popesko (1998) indican que estas relaciones también se observan en el perro. El nervio fibular no presentó divisiones en esta región. Por otra parte, el nervio tibial origina al nervio cutáneo sural caudal distal, el que se dirige hacia distal pasando caudalmente a las ramas musculares de la arteria y vena femoral caudal distal (Fig. 2).
En la cara medial de la región femoral, se observó el nervio femoral acompañado de la arteria y vena femoral, ordenados craneocaudalmente, cursan por la superficie medial del músculo pectíneo y vasto medial del cuádriceps, craneal al $\mathrm{m}$. gracilis y caudal al $\mathrm{m}$. sartorio. Se observó que el nervio femoral inerva directamente al músculo sartorio, al igual como se describe en el bovino (Gloobe, 1989). Sin embargo, en el perro este músculo es inervado por el nervio safeno (Evans). Aunque, Adams (1988) indica que en perros, el m. sartorio puede ser inervado tanto por el nervio femoral como el $\mathrm{n}$. safeno.También del $\mathrm{n}$. femoral emerge un ramo muscular el cual se divide enseguida en cuatro ramos para las respectivas cabezas del m. cuádriceps femoral. Finalmente origina al nervio safeno, el cual continúa hacia distal acompañado por la arteria y vena safena medial. En cuanto al nervio obturador se observó solo su porción extra pélvica, con un ramo al músculo gracilis y otro para el $\mathrm{m}$. aductor mayor y breve.

Región crural. En esta región se encuentra el nervio fibular común, el cual discurre entre el $\mathrm{m}$. bíceps femoral y la cabeza lateral del músculo gastrocnemio, a la cual atraviesa cerca de su margen craneal, profundizándose y localizándose entre los músculos gastrocnemio y sóleo, finalmente, se divide en un ramo profundo y uno superficial (Fig. 3). Una distribución similar sucede en el gato doméstico (Ghoshal, 1982) y también en el puma (Puma concolor), de acuerdo a lo observado en las imágenes obtenidas por Caro et al. (2013). El nervio fibular profundo inmediatamente se divide en tres ramos, dos ramos proximales que vuelven a divi-
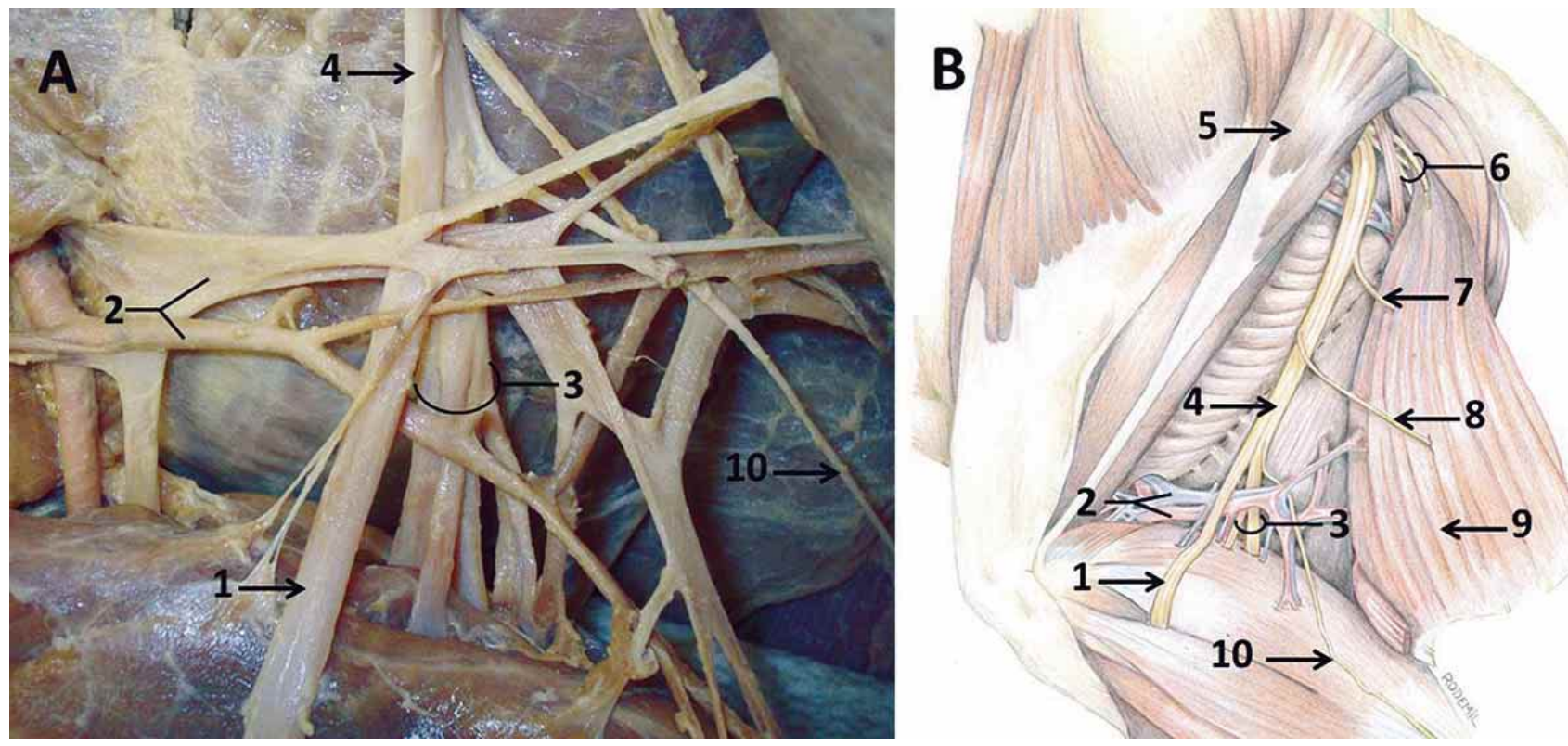

Fig. 2. A: Imagen de la región femoral distal y distribución del n. isquiático de león. B: Esquema del n. isquiático de león, Dibujo original Rodemil Medina. Miembro pélvico izquierdo, vista lateral. 1= N. fibular común. 2=Arteria y vena femoral caudal distal. 3= N. tibial. 4= N. isquiático. 5= M. glúteofemoral. 6= Ramos musculares a los músculos bíceps femoral, semitendinoso y semimembranoso.7= N. cutáneo sural caudal proximal. $8=\mathrm{N}$. cutáneo sural lateral. $9=\mathrm{M}$. bíceps femoral (rechazado caudalmente). 10= N. cutáneo sural caudal distal. 
dirse dando tres ramos para el músculo tibial craneal y tres para el extensor digital largo, y uno distal, que acompaña a la arteria y vena tibial craneal y se ubica entre los músculos fibular largo y extensor digital largo (Fig. 3). Para pasar finalmente a la región del pie cruzando entre el $\mathrm{m}$. extensor digital largo y el m. tibial craneal, profundamente al retináculo extensor. El nervio fibular superficial se dirige hacia distal superficialmente entre los músculos fibular largo y extensor digital lateral, en el tercio medio de la tibia pasa profundo al músculo fibular largo, emergiendo distalmente entre éste músculo y el extensor digital largo, pasando superficialmente al retináculo extensor.

Por caudal en la región crural se encuentra el nervio tibial, de este se origina un ramo para la cabeza medial del $\mathrm{m}$. gastrocnemio y un ramo para el $\mathrm{m}$. flexor digital superficial y m. sóleo. Cabe destacar que se observó que el músculo flexor digital superficial presenta un largo y grueso vientre, pero poco diferenciado de la cabeza lateral del gastrocnemio, a la que se adhiere estrechamente, esta característica anatómica también ha sido descrita por Barone (1967). Luego, el nervio tibial se introduce entre las dos cabezas del músculo gastrocnemio, donde proporciona un ramo para la cabeza lateral y un ramo para el músculo flexor digital profundo, finalmente se dirige hacia la región del pie.
El nervio cutáneo sural caudal distal se divide en dos ramos; uno pasa sobre el tendón calcáneo común y se ramifica en la región calcánea y el otro va a la superficie lateral del miembro e inerva la piel del tarso y metatarso, situación también descrita en el gato doméstico (Sisson \& Grossman, 1982).

El nervio safeno es subcutáneo y se dirige distocranealmente en la región crural en compañía de la arteria y vena safena medial.

Región del pie. El nervio tibial, al pasar por medial al hueso calcáneo, se divide en dos nervios plantares, uno medial y otro lateral. El nervio plantar medial recorre la superficie plantar del tendón del $\mathrm{m}$. flexor digital profundo y m. cuadrado plantar; este último músculo se observó como un tendón ancho y plano, lo cual concuerda con lo descrito por Barone. El n. plantar medial, pasa por medial al tendón del dedo II del $\mathrm{m}$. flexor digital superficial. Finalmente origina al nervio digital plantar común II y III, luego pasa en profundidad al torus metatarsal, para llegar a inervar los dedos como nervios digitales plantares propios (Fig. 4).

El nervio plantar lateral es más ancho y plano que el medial, y discurre entre el m. flexor digital breve y el tendón
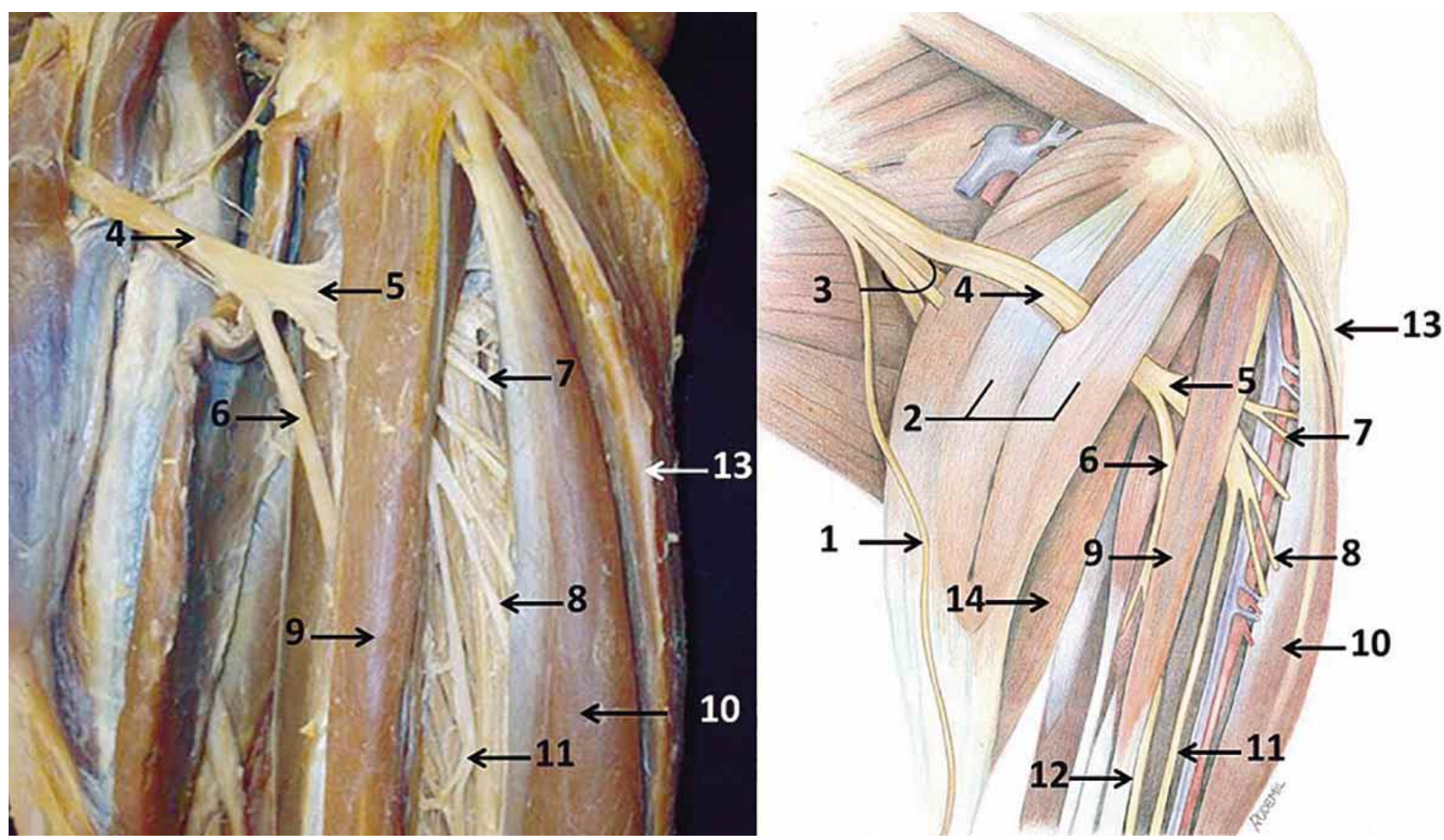

Fig. 3. Imagen y esquema de la región crural de león. Miembro pélvico derecho, vista lateral. Dibujo original Rodemil Medina. 1= N. cutáneo sural caudal distal. $2=$ Cabeza lateral del m. gastrocnemio. $3=\mathrm{N}$. tibial. $4=\mathrm{N}$. fibular común. $5=\mathrm{N}$. fibular profundo. $6=\mathrm{N}$. fibular superficial. 7= Ramos al m. tibial craneal. 8= Ramos al m. extensor digital largo. 9= M. fibular largo. 10= M. extensor digital largo. 11= Ramo distal del n. fibular profundo. 12= Ramo distal del n. fibular superficial. 13= M. tibial craneal. 14= M. sóleo. 
del m. flexor digital profundo, a nivel de la articulación tarsometatarsiana se divide en dos ramos al m. flexor digital breve, músculo que presentó gran desarrollo en el espéecimen disecado, en concordancia con lo descrito en el león por Barone y en el puma por Caro et al. El n. plantar lateral origina también un ramo profundo que se introduce entre los $\mathrm{m}$. interóseos, y un ramo que continua hacia los dedos como nervio digital común IV para inervar las superficies yuxtapuestas de los dedos IV y V (Fig. 4).

En la región dorsal del pie, el nervio fibular profundo forma los nervios digitales comunes dorsales, acompañados por las venas digitales comunes dorsales II, III, y IV, estos se dividen para formar los nervios digitales dorsales propios e inervar las superficies axiales y abaxiales de los dedos, acompañados por las venas digitales dorsales propias que provienen de las venas digitales comunes dorsales.
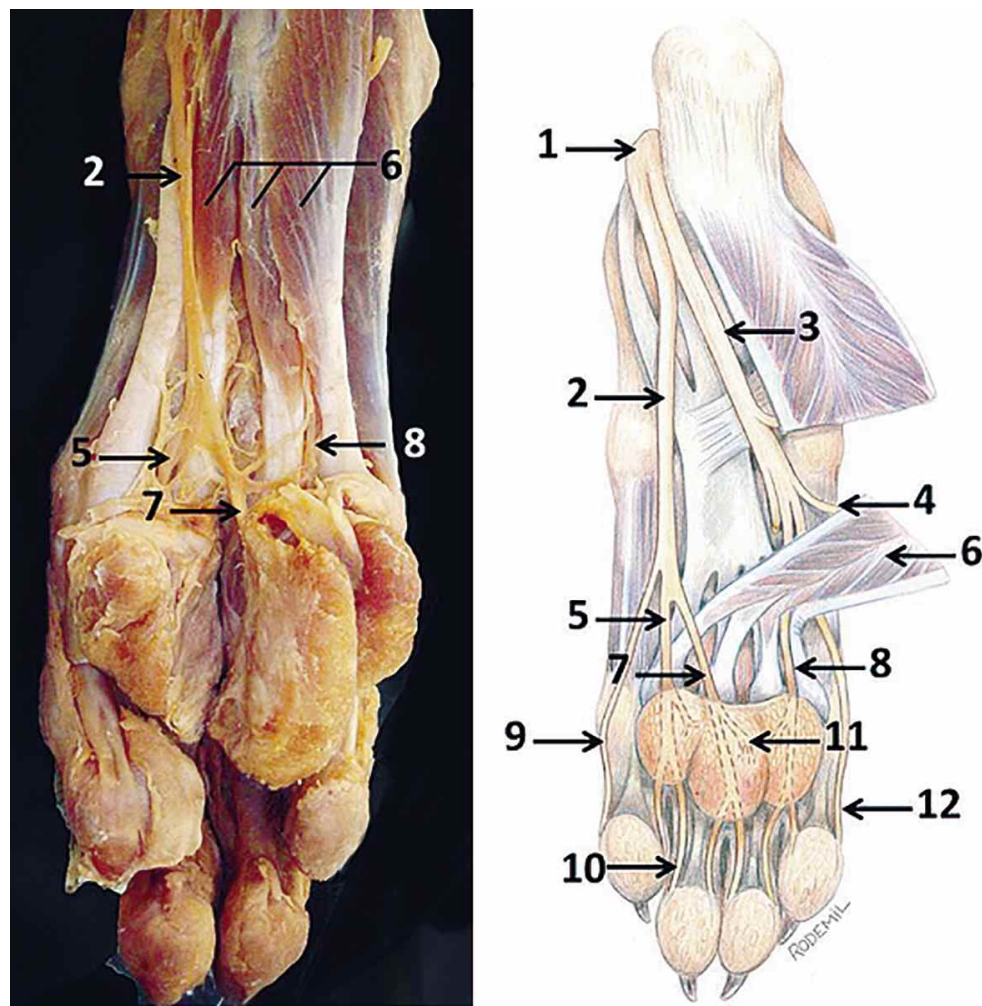

Fig. 4. Imagen y esquema de la región del pie de león. Miembro pélvico derecho, vista plantar. Dibujo original Rodemil Medina.1= N. tibial. 2= N. plantar medial. $3=\mathrm{N}$. plantar lateral. $4=$ Ramo al $\mathrm{m}$. flexor digital breve. $5=\mathrm{N}$. digital plantar común II. $6=$ M. flexor digital breve. $7=\mathrm{N}$. digital plantar común III. $8=$ N. digital plantar común IV. $9=$ N. digital plantar propio II abaxial.10= N. digital plantar propio III abaxial. 11= Ramos al torus metatarsal. 12= N. digital plantar propio $\mathrm{V}$ abaxial.

\section{CONCLUSIONES}

De acuerdo a la disección y estudio realizado, fue posible describir la inervación del miembro pélvico del león (Panthera leo). En cuanto a la inervación y su relación con el sistema músculo esquelético, los ramos musculares presentan gran desarrollo, permitiendo una fácil visualización. La diferenciación con la vasculatura es compleja solo en las estructuras de menor tamaño, pero la repleción arteriovenosa con látex y pigmento, facilita esta tarea. Según el estudio descriptivo de la inervación del miembro pélvico y sus relaciones topográficas musculares y vasculares, es posible mencionar que existe una gran similitud con lo descrito en el felino doméstico.

Este estudio permite ampliar el conocimiento anatómico referente a la inervación del miembro pélvico del león africano (Panthera leo), mostrando generalidades sobre las estructuras musculares y vasculares relacionadas topográficamente a esta, útil para los médicos veterinarios que se desempeñan en el área de fauna silvestre, para realizar procedimientos que requieran de información sobre la anatomía del león africano u otros grandes felinos.

MEDINA, P. R.; MORALES, M. P.; CONCHA, A. I. \& BORRONI, G. C. Anatomical description of pelvic limb innervation of African lion (Panthera leo). Int. J. Morphol., 32(3):889-894, 2014.

SUMMARY: The African Lion (Panthera $l e o$ ) belongs to the felidae family of the order carnivore, corresponding to large predators. In Chile it is found only in a state of captivity and is present in many zoos. As the concern and interest in these animals has increased, more complex procedures are performed each day. This generates the need for anatomical knowledge of this specie. This anatomical study was performed in order to describe the innervation of the pelvic limb of an African lion and topographical relationship with skeletal muscles and vascular structures. A corpse of an adult female lion, $130 \mathrm{~kg}$, was dissected and described by topographical regions. Proximal to distal: cingulum pelvic limb region, femoral region, crural region and foot region. Descriptions of each region were complemented by photographs and drawings. According to the descriptive study of the innervation of the pelvic limb, it was observed that there is a great similarity with that described in the domestic cat, excepting the great development of their nerves and muscular branches. This study provides information about the innervation of the pelvic limb of the African lion, showing the relation with muscular and vascular structures. The anatomical information provided in this study can be useful for medical procedures in this species and other big cats.

KEY WORDS: African lion; Ischiatic nerve; Innervation; Pelvic limb. 


\section{REFERENCIAS BIBLIOGRÁFICAS}

Adams, D. R. Anatomía canina: Estudio sistémico. Zaragoza, Acribia, 1988. pp.431-43.

Barone, R. La myologie du lion (Panthera leo). Mamm., 31(3):459514, 1967.

Caro, C.; Concha, I.; Borroni, C. \& Guerrero, J. Descripción anatómica de los músculos del miembro pélvico de puma (Puma concolor). Santiago, Universidad Santo Tomás, 2013. pp.5968 .

Done, S.; Goody, P.; Evans, S. \& Stickland, N. Atlas en color de anatomía veterinaria: El perro y el gato. Madrid, Elsevier, 2002. Cap. 10.

Evans, H. F. Miller's Anatomy of the dog. $4^{\text {th }}$ ed. St. Louis, Saunders, 2013. pp.636-56.

Ghoshal, N. Neurología de los carnívoros. En: Sisson, S. \& Grossman, J. D. Anatomía de los animales domésticos. $5^{\text {a }}$ ed. Barcelona, Salvat Editores, 1982. pp.1862-87.

Gloobe, H. Anatomía aplicada del bovino. San José, Instituto Interamericano de Cooperación para la Agricultura, 1989. pp.138.

Popesko, P. Atlas de anatomía topográfica de los animales domésticos. $2^{\mathrm{a}}$ ed. Barcelona, Masson, 1998. Tomo III. pp.36, 78, 177.

Sisson, S. \& Grossman, J. D. Osteología de los carnívoros. En: Sisson, S. \& Grossman, J. D. Anatomía de los animales domésticos. $5^{\text {a }}$ ed. Barcelona, Salvat Editores, 1982. pp.1571648 .

\author{
Dirección para Correspondencia: \\ Rodemil Medina Puentes \\ Escuela de Medicina Veterinaria \\ Facultad de Medicina Veterinaria y Recursos Naturales \\ Universidad Santo Tomás \\ CHILE
}

Email: rodemilmedina@gmail.com

Recibido: 24-03-2014

Aceptado: 30-05-2014 